\title{
Pericarditis por Mycobacterium tuberculosis multiresistente en un paciente con infección por VIH. Reporte de un caso clínico y revisión de la literatura
}

Martín Lasso B. y Jorge Pérez G.

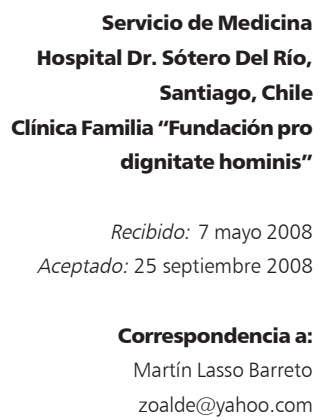

Correspondencia a: zoalde@yahoo.com

\section{Pericarditis due to multidrug resistant Mycobacterium tuberculosis in an HIV infected patient. Case report and review of literature}

Multidrug resistant Mycobacterium tuberculosis infection represents a new clinical challenge and it burdens threat. The association with HIV/AIDS makes very hard to treat these two co-infections. We present a patient of 30 years old whose HIV infection was diagnosed 4 years before, and acquired M. tuberculosis pericarditis without response to initial treatment because of a multidrug resistant TB strain. We describe the clinical management, outcome and final recovery of the patient. The epidemiology, diagnosis and treatment of TB pericarditis in HIV positive patients are reviewed.

Key words: Multidrug resistant tuberculosis; pericarditis tuberculous, HIV, AIDS.

Palabras clave: Tuberculosis multi-resistente, pericarditis tuberculosa, VIH, SIDA.

\section{Introducción}

$\mathrm{L}$ a combinación de infección por VIH y Mycobacterium tuberculosis representa un desafío terapéutico de gran relevancia. Las conocidas interacciones de rifampicina con los anti-retrovirales del tipo inhibidores de proteasa e inhibidores no nucleótidos de la transcriptasa reversa limitan el tratamiento concomitante efectivo de estas dos infecciones ${ }^{1}$. Se ha definido como M. tuberculosis multi-resistente (TBC-MR) a aquellas cepas con resistencia in vitro a isoniacida (HIN) y a rifampicina (RFP) y $M$ tuberculosis de extrema resistencia (TBC-ER) a aquellas con resistencia in vitro a HIN y RFP dentro de los fármacos de primera línea y resistencia a cualquier fluoroquinolona y al menos un agente parenteral dentro de los fármacos de segunda línea². Según datos de la OMS para el período 2002-2007 se reportaron en Latinoamérica 349.278 casos de tuberculosis (TBC), 12.070 de los cuales eran TBC-MR. En Chile, en igual período se diagnosticaron 2.599 casos de TBC de los cuales 24 eran TBC-MR. La incidencia de TBC para el año 2006 en Chile fue de 15 casos por cada 100.000 habitantes/año de los cuales sólo $0,7 \%$ fueron TBCMR y ha sido reportado un solo caso de TBC-ER ${ }^{3,4}$. Por otro lado, las localizaciones extra-pulmonares de la TBC, y particularmente la pericárdica, son siempre graves. La TBC pericárdica adopta un curso clínico subagudo que puede conducir a la insuficiencia cardíaca o incluso al taponamiento pericárdico. Su tratamiento es prolongado, requiere apoyo de corticosteroides y procedimientos quirúrgicos, evolucionando, pese a todo ello, con secuelas crónicas restrictivas e incluso letales ${ }^{5}$. No se han comunicado en la literatura médica nacional casos de pericarditis tuberculosa causadas por un agente multi-resistente y los reportes al respecto en la literatura internacional son anecdóti$\cos ^{6,7}$. En este contexto este caso de pericarditis por TBC-MR, ocurrido en nuestro centro hace más de nueve años, representa un problema de importancia y actualidad.

\section{Caso Clínico}

Paciente con 30 años de edad, sexo masculino, homosexual, portador de VIH confirmado por el ISP en marzo de 1995, con antecedente de candidiasis orofaríngea y un recuento de linfocitos CD4 (L-CD4) de 91 céls $/ \mathrm{mm}^{3}$ (15/01/1998), en tratamiento anti-retroviral (TAR) con zidovudina, lamivudina e indinavir, iniciado un año antes de su ingreso, en enero de 1999. Fue admitido en nuestro hospital por un cuadro de fiebre y disnea, con evolución de 10 días. En ese momento tenia un recuento de L-CD4 de 314 céls $/ \mathrm{mm}^{3}$ y una carga viral indetectable. En su examen físico destaca- 
ban lesiones en la pierna derecha compatibles con herpes zoster y un frote pericárdico. La radiografía de tórax demostró cardiomegalia (Figura 1), el electrocardiograma un supra-desnivel ST con inversión de T y el ecocardiograma de superficie una efusión pericárdica importante con disfunción sistólica ventricular leve. La punción percutánea del pericardio dio salida a un líquido cetrino-hemorrágico con 2.900 céls $/ \mathrm{mm}^{3}, 74 \%$ de mononucleares, hematíes $(+++)$, glucosa de $63 \mathrm{mg} /$ $\mathrm{dL}$, proteínas $4,3 \mathrm{~g} / \mathrm{dL}$, y adenosin deaminasa (ADA) de 67 U/L. Dos baciloscopias (BK) del líquido pericárdico fueron negativas. Se decidió el inicio empírico de tratamiento anti-tuberculoso con HIN 300 mg/día, RFP $600 \mathrm{mg} /$ día, pirazinamida (PZ) $1.500 \mathrm{mg} /$ día y etambutol (E) $1.200 \mathrm{mg} /$ día. La TAR se suspendió por la interacción de indinavir con RFP. Al noveno día de hospitalización se realizó una biopsia pericárdica que demostró una inflamación mononuclear granulomatosa sin zonas de necrosis y con presencia de bacilos ácidoalcohol resistentes (BAAR). El estudio citológico del líquido pericárdico no detectó la presencia de células neoplásicas. La prueba de PPD fue negativa. Persistió febril luego de 12 días de terapia por lo que se amplió la cobertura anti-micobacteriana con claritromicina 1.000 $\mathrm{mg}$ /día y amikacina $1.000 \mathrm{mg}$ /día lográndose un descenso de la fiebre tres días después. El paciente permaneció un total de 20 días hospitalizado y dado su estabilidad se trasladó a un centro de menor complejidad para continuar su tratamiento anti-tuberculoso.

Se recibió resultado de la reacción de polimerasa en cadena (RPC) para M. tuberculosis complex positiva en líquido pericárdico (Laboratorio de la Pontificia Universidad Católica) por lo que se decidió ajustar esquema a HIN + RFP + PZ + E por un mes y amikacina durante 14 días, a la vez que se suspendió la claritromicina. Luego, para el segundo mes se suspendió RFP quedando con HIN + PZ + E y ciprofloxacina (Cp) 1.000 $\mathrm{mg} /$ día, en dosis diarias, y se reinicio la TAR con el mismo esquema inicial. Evolucionó favorablemente, sin fiebre, y luego de completar 51 dosis de tratamiento anti-tuberculoso diario fue enviado a su domicilio con esquema HIN: $800 \mathrm{mg}$ /día y RFP: $600 \mathrm{mg}$ /día bisemanal. El cultivo de liquido pericárdico confirmado por ISP demostró un $M$. tuberculosis.

Ambulatoriamente el paciente siguió recibiendo TAR y permaneció en condición estable hasta la dosis 28 de la fase bisemanal, momento en que reapareció la fiebre y el dolor toráxico por lo que debió ser rehospitalizado (cuatro meses después de su egreso). El reporte de sensibilidad del ISP para $M$ tuberculosis aislado en el líquido pericárdico demostró resistencia a HIN y RFP. Se inició tratamiento con kanamicina (K) $750 \mathrm{mg} /$ día IM, etionamida (Et) $750 \mathrm{mg} /$ día, PZ 1.250 $\mathrm{mg} /$ día y E $1.000 \mathrm{mg} /$ día, esquema que recibió durante ocho semanas. A la cuarta semana de tratamiento con esta nueva terapia el paciente presentó un episodio de crisis convulsiva tónico-clónico generalizada que se manejó con fenitoína. Una TAC de cerebro y estudio de LCR fueron normales y el evento se atribuyó a reacción adversa causada por Et. Fue tratado con fenitoína y no presentó nuevas crisis. Al completar las ocho semanas de tratamiento se suspendió la $\mathrm{K}$ y se mantuvo con PZ + E + Et durante 10 semanas más. En este lapso persistió con alzas térmicas aisladas. En la semana 12 de tratamiento con esquema de segunda línea presentó un dolor toráxico bajo; se evidenció una colección subxifoídea que fue puncionada dando salida a material purulento con presencia de BAAR. El cultivo a los 30 días demostró incontables colonias de M. tuberculosis. Se mantuvo el tratamiento sin variaciones y curaciones, pese a lo cual persistió febril. En la semana 18 se le practicó un ecocardiograma que demostró una colección retroesternal con presencia de efusión pericárdica en moderada cuantía; se realizó drenaje quirúrgico de la colección con salida de abundante líquido purulento, también con BAAR, por lo que se decidió agregar nuevamente $\mathrm{Cp} 1.000 \mathrm{mg}$ /día a su esquema de $\mathrm{PZ}+\mathrm{E}+\mathrm{Et}$ recibiendo tratamiento diario, de este modo, por 22 semanas más. El cultivo para micobacterias de la colección retroesternal fue negativo. En la semana 26 desarrolló un absceso ganglionar cervical derecho que fue drenado y demostró BAAR, con cultivo de Koch también negativo.

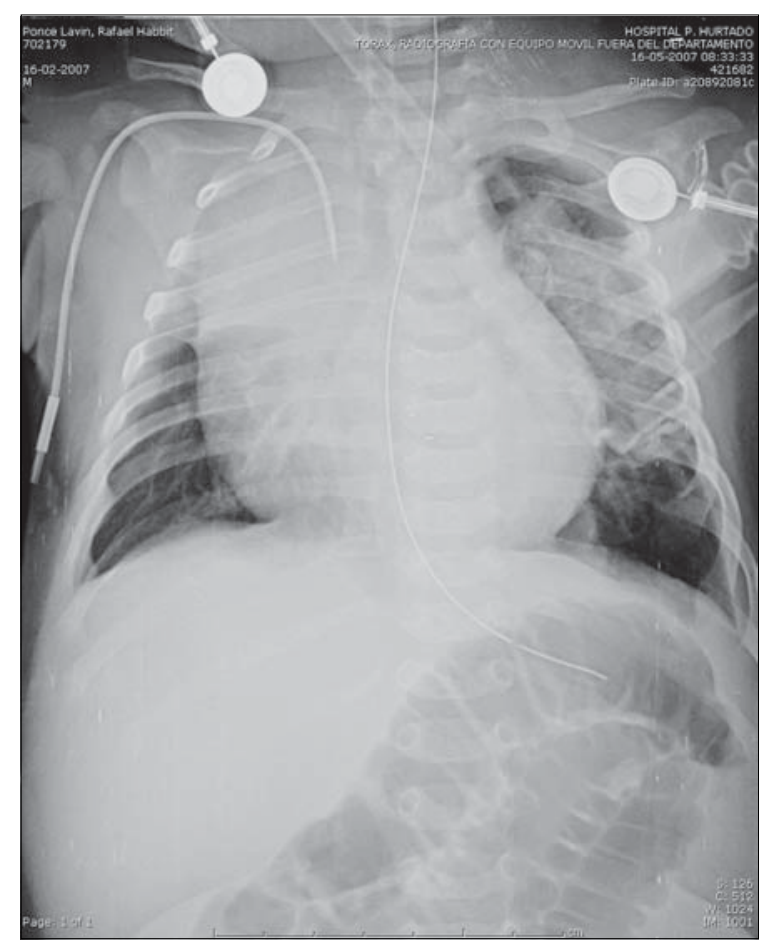

Figura 1. Rx tórax AP en que destaca aumento de la silueta cardiaca (ICT: 60\%), en paciente con pericarditis purulenta. Además imágenes de relleno alveolar a izquierda. CVC por vena subclavia derecha, tubo endotraqueal y sonda nasogástrica bien ubicados. 
Tabla 1. Correlato temporal entre manifestaciones clínicas, microbiología, tratamiento anti-retroviral y tratamiento anti-tuberculoso

\begin{tabular}{|c|c|c|c|c|c|c|c|c|c|c|}
\hline & \multicolumn{10}{|c|}{ Día de evolución } \\
\hline & $1-12$ & $13-20$ & $21-28$ & 29-51 & $52-172$ & $173-229$ & $230-258$ & $259-300$ & $301-454$ & $455-608$ \\
\hline \multirow{2}{*}{$\begin{array}{l}\text { Condición } \\
\text { clínica }\end{array}$} & Fiebre & \multirow[t]{2}{*}{ Afebril } & \multirow[t]{2}{*}{ Afebril } & \multirow[t]{2}{*}{ Afebril } & Fiebre, dolor & \multirow[t]{2}{*}{ CTCG } & Absceso & Absceso & Absceso & Alta \\
\hline & Disnea Frote & & & & toráxico* & & sub-xifoideo & retro-esternal & ganglio cervical & Sin fiebre \\
\hline \multirow[t]{2}{*}{ MIB } & BK (-) Bp & \multirow[t]{2}{*}{ No } & \multirow[t]{2}{*}{$\operatorname{RPC}(+)$} & \multirow[t]{2}{*}{ No } & $\mathrm{CT}(+)$ & \multirow[t]{2}{*}{ No } & $\mathrm{BK}(+)$ & $\mathrm{BK}(+)$ & $\mathrm{BK}(+)$ & \multirow[t]{2}{*}{ No } \\
\hline & con BK $(+)$ & & & & L. Pericárdico & & $\mathrm{CT}(+)$ & $C \mathrm{CT}(-)$ & $C T(-)$ & \\
\hline TAR & No & No & No & Sí & Sí & Sí & Sí & Sí & Sí & Sí \\
\hline \multirow[t]{2}{*}{ T-TBC } & \multirow[t]{2}{*}{ HIN-RFP-PZ-E } & HÍN-RFP-PZ- & HIN-RFP- & \multirow[t]{2}{*}{ HIN-PZ-E-Cp } & \multirow[t]{2}{*}{ HIN-RFP** } & \multirow[t]{2}{*}{ PZ-E-Et-K } & \multirow[t]{2}{*}{ PZ-E-Et } & \multirow[t]{2}{*}{ PZ-E-Et } & \multirow[t]{2}{*}{ PZ-E-Et-Cp } & \multirow[t]{2}{*}{ PZ-E-Et-Cp } \\
\hline & & E-AMK-Clar & PZ-E-AMK & & & & & & & \\
\hline
\end{tabular}

Luego de completar 40 semanas de tratamiento de segunda línea en el hospital, fue dado de alta sin fiebre y con una ecocardiografía que descartó la presencia de colecciones retroesternales. En una fase ambulatoria recibió esquema con $\mathrm{PZ}+\mathrm{E}+\mathrm{Et}+\mathrm{Cp}$ diario durante 22 semanas más. En suma llego a recibir 62 semanas de tratamiento anti-tuberculoso de segunda línea (Tabla 1). En la actualidad, nueve años después, paciente se encuentra haciendo vida normal y en TAR.

\section{Discusión}

La pericarditis tuberculosa es un infección inhabitual, encontrándose en $1 \%$ de las autopsias por TBC de cualquier localización y en 1 a $2 \%$ de las TBC pulmonares. En la actualidad tiene una marcada relación con la infección por VIH y, al igual que la mayoría de formas extra-pulmonares, se presenta especialmente en etapa SIDA encontrándose esta asociación hasta en $50 \%$ de las pericarditis TBC en ciertas áreas de África ${ }^{8,9}$.

La TBC es una enfermedad marcadora de SIDA; no obstante, como fue el caso de nuestro paciente, no sólo aparece en condiciones de inmunosupresión grave sino que se pueden encontrar en cualquier nivel de L-CD4. Las manifestaciones clínicas y de imagen de la pericarditis tuberculosa no difieren entre pacientes portadores respecto de no portadores de VIH; sin embargo, la formación de granulomas se ve limitada en los pacientes con infección por VIH cuando tienen recuentos bajos de L-CD49.

En $80 \%$ de los casos el líquido pericárdico de la TBC es un exudado hemorrágico con predominio mononuclear, proteínas elevadas y glucosa muy baja. Las BK pueden ser positivas en 0 a $42 \%$ de las veces, los cultivos entre 53 y $75 \%$ y el estudio anátomo-patológico de la biopsia de pericardio tiene una sensibilidad de
10 a $64 \%$ para el diagnóstico de $\mathrm{TBC}^{10}$. En nuestro paciente llamó la atención una glucosa en líquido pericárdico en valor normal. El ADA es una herramienta ampliamente útil en el diagnóstico de TBC pericárdica con un punto de corte establecido sobre 35 UI. Un meta-análisis reciente detectó una sensibilidad de $88 \%$, especificidad de $83 \%$, valor predictor positivo de $83 \%$ y valor predictor negativo de $88 \%$ para la prueba de ADA en el diagnóstico de TBC pericárdica ${ }^{11}$; independiente de aquello, la presencia de hematíes en el líquido pericárdico hace difícil su interpretación ya que puede elevarla falsamente.

En la pericarditis tuberculosa el diagnóstico se certifica con la anatomía patológica clásica y con el cultivo de líquido o tejido pericárdico; este segundo demora entre uno y dos meses. Las técnicas de RPC para $M$. tuberculosis no han sido una solución en este aspecto por su pobre sensibilidad en fluidos, fácil contaminación y presencia de falsos positivos ${ }^{10,12}$.

En la época en que ocurrió este caso, nuestro centro administraba TAR consistente en zidovudina, lamivudina e indinavir, la misma que se suspendió durante el primer mes de tratamiento anti-tuberculoso por la interacción de indinavir con RFP pero, dada la buena respuesta inicial, se decidió modificar la terapia antituberculosa para el segundo mes suspendiendo la RFP y agregando $\mathrm{Cp}$ para poder reintroducir la TAR. En la actualidad se ha logrado definir que el $\mathrm{Cp}$ no es una buena opción para sustitución de regímenes de primera línea en el caso de la TBC pulmonar con cultivo positivo, dada la alta incidencia de recaídas y la demora en la negativización de la expectoración en estos pacientes ${ }^{13}$. Las guías clínicas actuales del CDC no incluyen a $\mathrm{Cp}$ como un fármaco útil para el tratamiento de la $\mathrm{TBC}^{14}$.

Inicialmente el paciente presentó una mejoría clínica pero la reaparición de fiebre y dolor toráxico, luego 
de haber completado su fase diaria y 28 dosis de tratamiento bisemanal, representó una alerta respecto de la presencia de una cepa resistente de $M$. tuberculosis; no se consideró la posibilidad de una reconstitución inmune dado que el paciente había recibido más de cinco meses de TAR e inicialmente tenía L-CD4 $>300$ céls $/ \mathrm{mm}^{3}$. Es conocido que los pacientes con TBC pericárdica fallecen si no son tratados en un lapso de cuatro meses, y que incluso, en los grupos tratados, la mortalidad es de hasta $40 \%{ }^{15}$. Por ello, la demora en obtener cultivos positivos para $M$. tuberculosis y la latencia posterior hasta definir la susceptibilidad respectiva del agente, es un problema. En la actualidad se trabaja en métodos para la detección rápida de cepas resistentes como el ensayo de observación microscópica de diagnóstico de TBC y susceptibilidad a fármacos (MODS en inglés: microscopic observation drug susceptibility) que fuera aplicado en muestras de expectoración cultivadas en medios líquidos en tres cohortes peruanas con diverso nivel de riesgo de cepas multi-resistentes y que tardó en determinar la existencia de TBC y la susceptibilidad de la cepa a HIN, RFP, E y estreptomicina en promedio siete días, con $97,8 \%$ de sensibilidad en el diagnóstico del agente ${ }^{16}$, o como la prueba basada en fagos para la detección de cepas resistentes a RFP: FastPlaqueresponse $^{\circledR}$ (Bistec Laboratorios LTDA. Ipswich, UK) con una sensibilidad de $95,4 \%$, especificidad de $97,2 \%$ y una precisión global de $96,5 \%$ respecto del estándar de oro que es el cultivo con el antibiograma; esta prueba se aplica directamente en la tinción de BAAR y su resultado se obtiene en 48 horas a diferencia de los 28 a 85 días que tardan los métodos clásicos ${ }^{17}$.

Dentro del esquema de segunda línea utilizado una vez que se confirmó la TBC-MR se incluyó la Et, fármaco asociado a crisis convulsivas ${ }^{18} \mathrm{y}$ al que fue atribuido el evento convulsivo de nuestro paciente, habiéndose descartado razonablemente otro origen. Este episodio se manejó con fenitoína y no fue necesaria la suspensión del fármaco. Kanamicina fue utilizada por ocho semanas, es decir 56 dosis y luego se continuó con tres fármacos activos $(\mathrm{PZ}+\mathrm{E}+\mathrm{Et})$, de acuerdo a la recomendación de las normas del MINSAL para tratamiento de la TBC vigentes en esa época ${ }^{19}$. A este tratamiento se agregó luego $\mathrm{Cp}$ por persistencia de baciloscopias positivas en las lesiones ganglionares satélites. Esta persistencia del agente en un paciente bajo tratamiento anti-TBC debe ser juzgada con prudencia ya que la mayoría de las veces no representa una micobacteria resistente activa e incluso pueden tratarse de BAAR no viables. Al Moamary y cols ${ }^{20}$, demostraron en una serie de 428 pacientes con TBC pulmonar y cultivos positivos iniciales que en $7 \%$ las baciloscopias continúan siendo positivas pasadas las
20 semanas de tratamiento y que en ese grupo, $77 \%$ de los pacientes presentan cultivos negativos.

Si bien se ha establecido que el uso de corticosteroides disminuye la mortalidad, la necesidad de drenajes pericárdicos y los problemas ulteriores derivados de la constricción pericárdica, en nuestro paciente no se indicó corticoterapia ya que en la época en que se enfrentó el caso ésta no era una práctica establecida en nuestro medio e, incluso en la actualidad, porcentajes cercanos al $50 \%$ de los médicos clínicos que atienden en las áreas de mayor incidencia de TBC pericárdica, como el África subsahariana o algunos países asiáticos, no los utilizan ${ }^{15,21,22}$. Pese a todo aquello, la recomendación actual, basada en la evidencia, es que al igual que en la meningitis TBC, la pericarditis debe ser manejada con corticosteroides por un lapso de al menos seis meses ${ }^{14}$.

Hoy en día, la mayoría de las guías clínicas recomiendan para el tratamiento de TBC-MR el uso de PZ, E, una fluoroquinolona, un agente parenteral, con o sin un agente alternativo como: Et, cicloserina, ácido p-aminosalicílico, claritromicina, amoxicilina/ácido clavulánico o linezolid. El período de tratamiento debe extenderse por 18 a 24 meses de dosis diarias ${ }^{14,23,24}$ (Tablas 2 y 3). Este tiempo no se pudo completar en nuestro paciente pero, pese a ello, su TBC pericárdica no se reactivó. Por último, en un análisis retrospectivo de Chan y cols ${ }^{25}$, la curación de pacientes con TBCMR adecuadamente tratados alcanza a $75 \%$ y la mortalidad atribuible es de $12 \%$, demostrando de paso, que el uso de fluroquinolonas dentro del tratamiento es un factor favorable en el pronóstico. En particular, moxifloxacina parece ser la mejor opción dada su mayor actividad in vitro ${ }^{26}$, su demostrada actividad bactericida en modelos animales ${ }^{27}$ y su temprano efecto bactericida en expectoración en pacientes con $\mathrm{TBC}^{28,29}$.

Este complejo caso nos demuestra que un paciente infectado por una TBC-MR puede tener una buena respuesta inicial al tratamiento clásico pero ante una evolución posterior no esperada se debe sospechar la presencia de cepas resistentes siendo fundamental disponer siempre del estudio de susceptibilidad del agente aislado. Una vez ajustado el tratamiento, es necesario mantenerlo pese a eventuales complicaciones asociadas a la infección de base o frente a baciloscopias positivas intra-tratamiento. Por último, si bien existen opciones terapéuticas para el manejo de la TBC-MR debemos realizar un esfuerzo para evitar su diseminación, básicamente con la vigilancia del cumplimiento del tratamiento en los pacientes portadores de TBC no resistentes.

Agradecimientos. Los autores agradecen la desinteresada colaboración de Inés Cerón en la revisión crítica de este trabajo. 


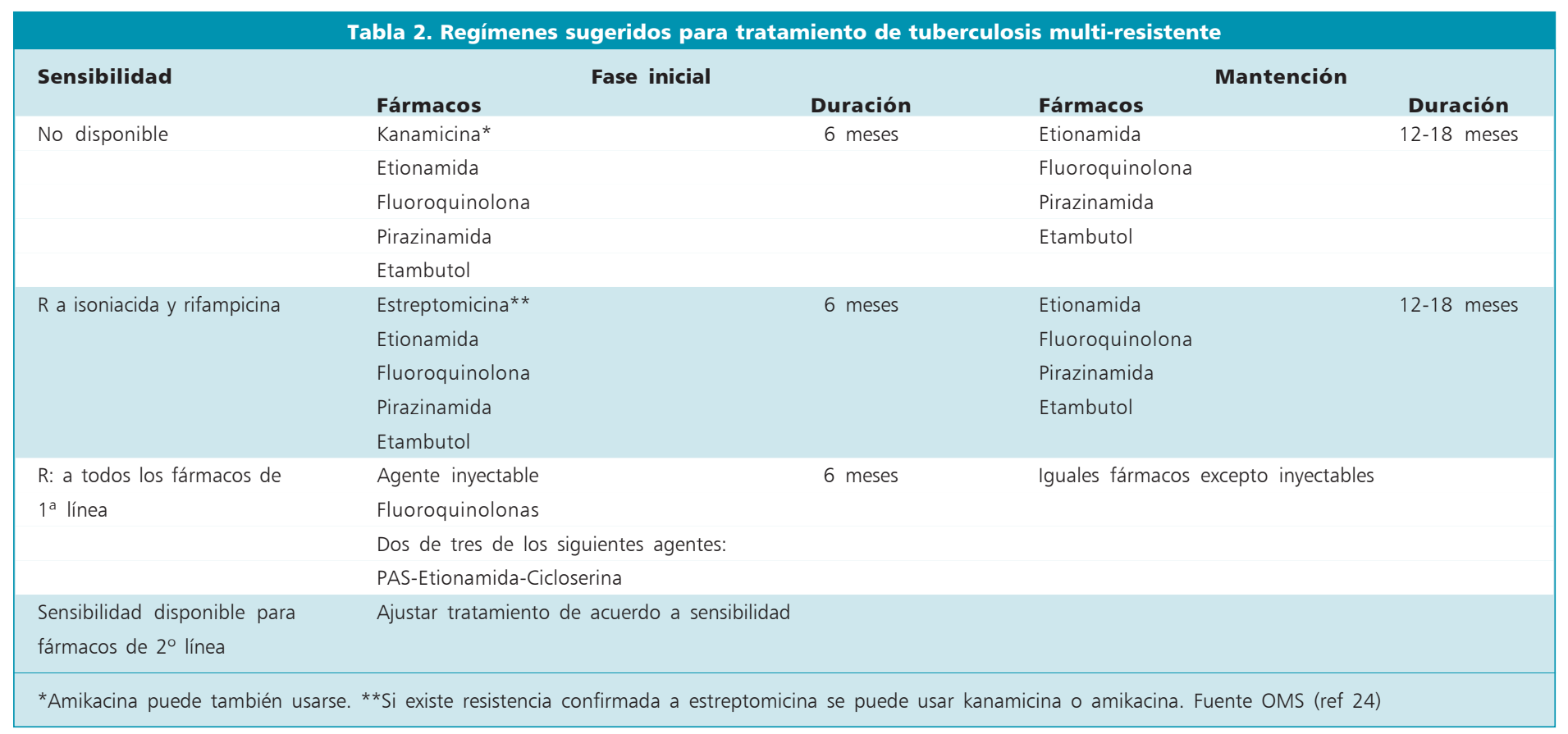

\begin{tabular}{|c|c|c|c|c|}
\hline \multirow{2}{*}{ Fármaco } & \multirow{2}{*}{$\begin{array}{l}\text { Mecanismo } \\
\text { de acción }\end{array}$} & \multicolumn{3}{|c|}{ Dosis diaria recomendada* } \\
\hline & & $\begin{array}{l}\text { Dosis } \\
\mathrm{mg} / \mathrm{kg}\end{array}$ & $\begin{array}{c}\text { Mínima } \\
\text { (mg) }\end{array}$ & $\begin{array}{c}\text { Máxima } \\
\text { (mg) }\end{array}$ \\
\hline Amikacina & Bactericida & 15 & 750 & 1.000 \\
\hline Capreomicina & Bactericida & 15 & 750 & 1.000 \\
\hline Ciprofloxacina & Bactericida & $10-20$ & 1.000 & 1500 \\
\hline Cicloserina & Bacteriostática & $10-20$ & 500 & 750 \\
\hline Etionamida & Bactericida & $10-20$ & 500 & 750 \\
\hline Kanamicina & Bactericida & 15 & 750 & 1.000 \\
\hline Ofloxacina & Bactericida & $7,5-15$ & 600 & 800 \\
\hline Ácido p-aminosalicílico & Bacteriostático & 150 & $8 \mathrm{~g}$ & $12 \mathrm{~g}$ \\
\hline Protionamida & Bactericida & $10-20$ & 500 & 750 \\
\hline
\end{tabular}

\section{Resumen}

La infección por Mycobacterium tuberculosis multiresistente representa una nueva y grave amenaza. La asociación con infección por VIH/SIDA vuelve muy complejo el tratamiento exitoso de ambas infecciones. Presentamos el caso de un paciente de 30 años de edad, con cuatro años de diagnóstico de infección por VIH, que desarrolló una pericarditis por $M$ tuberculosis sin una respuesta favorable inicial al tratamiento debido a que se trataba de una cepa multi-resistente. Se describe el manejo médico, evolución y recuperación final del paciente. Se revisa la epidemiología, diagnóstico y tratamiento de la pericarditis $\mathrm{TBC}$ en pacientes con infección por VIH.

\section{Referencias}

1.- Sande M A, Eliopoulos G, Moellering R, Gilbert D, Pavia A. Drug/drug interactions: Antiretroviral drugs \& drugs used in treatment of HIV-associated infections \& malignancies. En The Sanford Guide to HIVAIDS Therapy 2006-2007 15th Ed. 2007: 156-61.

2.- Raviglione, M, Smith, I. XDR Tuberculosisimplications for global public health. N Engl
J Med 2007; 356: 656-9.

3.- Informe de OMS sobre la epidemiología de la tuberculosis en Chile año 2006 www.who.int/ GlobalAtlas/predefinedReports/TB/ PDF_Files/chl.pdf (Accedido: 20 marzo 2008)

4.- Anti-tuberculosis drug resistance in the world. Fourth Global Report en http:// www.who.int/tb/publications/2008/ drs_report4_26Feb08_pdf Ingreso (Accedido: 29 abril 2008).
5.- Fowler NO. Tuberculous pericarditis. JAMA 1991; 266: 99-103.

6.- Mastroianni A, Coronado O, Chiondo F. Tuberculous pericarditis and AIDS: Case reports and review. Eur J Epidemiol 1997; 13: 755-9.

7.- Horn D L, Hewlett D Jr, Alfalla C, Peterson S. Fatal hospital acquired multidrug resistant tuberculous pericarditis in two patients with AIDS. N Eng J Med 1992; 327: 1816-7. 
8.- Reuters H, Burgués L J, Doubell A F. Epidemiology of pericardial effusions at a large academic hospital in South Africa. Epidemiol Infect 2005; 133: 393-9.

9.- Mayosi B M, Burgués L J, Doubell A F. Tuberculous pericarditis. Circulation 2005; 112: 3608-16.

10.- Cegielski J P, Devlin B H, Morris A J, Kitinya J N, Pulipaka U P, Lema L E et al. Comparison of PCR, culture and histopathology for diagnosis of tuberculous pericarditis. J Clin Microbiol 1995; 35: 3254-7.

11.- Tuon F F, Litvoc M N, Lopes M I. M. Adenosine deaminase and tuberculous pericarditis-- a systematic review with metaanalysis. Acta Trop 2006; 99: 67-74

12.- Lee J H, Lee C W, Lee S G, Yang H S, Hong M K, Kim J J, et al. Comparison of polymerase chain reaction with adenosine deaminase activity in pericardial fluid for the diagnosis of tuberculous pericarditis. Am J Med 2002; 113: 519-21.

13.- Ziganshina L, Vizel A, Squire S. Fluoroquinolones for treating tuberculosis. Cochrane Database Syst Rev 2005; 3:CD004795.

14.- American Thoracic Society, CDC and Infectious Diseases Society of America. Treatment of tuberculosis. http:// www.cdc.gov/mmwr/PDF/rr/rr52II.pdf. (Accedido: 01-mayo-2008).

15.- Yang C C, Lee M H, Liu J W, Leu H S. Diagnosis of tuberculous pericarditis and treatment without corticosteroid in a tertiary teaching hospital in Taiwan: a -14 years experience. J Microbiol Immunol
Infect 2005; 38: 47-52

16.- Moore D A, Evans C, Gilman R, Caviedes L, Coronel J, Vivar A et al. Microscopicobservation drug susceptibility assay for the diagnosis of TB. N Eng J Med 2006; 335: 1539-50

17.- Albert H, Trollip A P, Seaman T, Abrahams C, Mole R J, Jordaan A, et al. Evaluation of a rapid screening test for rifampicin resistance in re-treatment tuberculosis patients in the Eastern Cape. S Afr Med J 2007; 97: 858-63.

18.- Snavely S C, Hodges G R. The neurotoxicity of antibacterial agents. Ann Intern Med 1984; 101: 92-104.

19. - Normas de Tratamiento de la Tuberculosis en Actualización Normas Técnicas. Programa Nacional de Control de la Tuberculosis. Departamento EpidemiologíaDivisión Programas de Salud. 1996: 81-7.

20.- Al Moamary M S, Black W, Bessuille E, Elwood K, Vedal S. The significance of the persistent presence of acid-fast bacilli in sputum smears in pulmonary tuberculosis. Chest 1999; 116: 726-31.

21.- Strang J I, Nunn A J, Johnson D A, Casbard A, Gibson D G, Girling D J. Management of tuberculous constrictive pericarditis and tuberculous pericardial effusion in Transkei: results at 10 years follow up. QJM 2004; 97: 525-35.

22.- Wiysonge C S, Ntsekhe M, Gumedze F, Sliwa K, Blackett K N, Commerford P J, et al. Contemporary use of adjunctive corticosteroids in tuberculous pericarditis. Int J Cardiol 2008; 124: 388-90.

23.- Normas de Tratamiento de la Tuberculosis en Actualización Normas Técnicas. Programa Nacional de Control de la Tuberculosis. Departamento EpidemiologíaDivisión Programas de Salud. MINSAL 2005.

24.- OMS Guías de tratamiento de tuberculosis multiresitente: http://www.who.int/tb/ publications/cds_tb2003_313/en/index.html (Accedido: 29-abril-2008).

25.- Chan E, Laurel V, Strand M, Chan J, Huynh M L, Globe M, et al. Treatment and outcome analysis of 205 patients with multidrug-resistant tuberculosis. Am J Respir Crit Care Med 2004; 169: 1103-9.

26.- Hu Y, Coates A R, Mitchison D A. Sterilizing activities of fluoroquinolones against rifampin-tolerant populations of Mycobacterium tuberculosis. Antimicrob Agents Chemother 2003; 47: 653-7.

27.- Veziris N, Truffot-Pernot C, Aubry A, Jarlier V, Lounis N. Fluoroquinolonecontaining third-line regimen against Mycobacterium tuberculosis in vivo. Antimicrob Agents Chemother 2003; 47: 3117-22.

28.- Gosling R D, Uiso LO, Sam N E, Bongard E, Kanduma EG, Nyindo M, et al. The bactericidal activity of moxifloxacin in patients with pulmonary tuberculosis. Am J Respir Crit Care Med 2003; 168: 1342-5.

29.- Pletz M W, De Roux A, Roth A, Neumann K H, Match H, Lode H. Early bactericidal activity of moxifloxacin in treatment of pulmonary tuberculosis: a prospective, randomized study. Antimicrob Agents Chemother 2004; 48: 780-2. 